\title{
Scale invariance of entanglement dynamics in Grover's quantum search algorithm
}

\author{
M. Rossi, ${ }^{1, *}$ D. Bruß, ${ }^{2}$ and C. Macchiavello ${ }^{1}$ \\ ${ }^{1}$ Dipartimento di Fisica and INFN-Sezione di Pavia, Via Bassi 6, 27100 Pavia, Italy \\ ${ }^{2}$ Institut für Theoretische Physik III, Heinrich-Heine-Universität Düsseldorf, D-40225 Düsseldorf, Germany
}

\begin{abstract}
We calculate the amount of entanglement of the multiqubit quantum states employed in the Grover algorithm, by following its dynamics at each step of the computation. We show that genuine multipartite entanglement is always present. Remarkably, the dynamics of any type of entanglement as well as of genuine multipartite entanglement is independent of the number $n$ of qubits for large $n$, thus exhibiting a scale invariance property. We compare this result with the entanglement dynamics induced by a fixed-point quantum search algorithm. We also investigate criteria for efficient simulatability in the context of Grover's algorithm.
\end{abstract}

\section{INTRODUCTION}

Although it is well-known that entanglement represents an essential ingredient in quantum communication and information, its role in the speed-up of quantum computational processes is not yet fully understood and still represents a debated question [1-4]. In particular, it is of great interest to investigate the role of multipartite entanglement in quantum algorithms. In Shor's algorithm multipartite entanglement was proved to be necessary to achieve exponential computational speed-up with quantum resources 1. Moreover, more recently it was shown that multipartite entangled states are employed in the Deutsch-Jozsa algorithm and in the first step of the Grover algorithm [5. In our work we investigate in detail the entanglement properties in the Grover algorithm [6], namely we study the behaviour of entanglement of the states of $n$ qubits along the whole computational process, and disclose in particular a noteworthy scale invariance property of its dynamics in terms of the geometric measure of entanglement (GME). Previous works on the entanglement dynamics in Grover's algorithm considered other entanglement measures and focused only on bipartite entanglement (see, for example, [7, 8]). We also study the entanglement dynamics in the fixed-point $\pi / 3$ quantum search algorithm 9, 10, and show that it turns out to be qualitatively similar to the Grover case.

This paper is organised as follows. In Sect. II we consider the Grover quantum search algorithm and study its entanglement dynamics for any number of qubits in the cases of one and two solutions to the search problem. In Sect. III we compare these results with the entanglement dynamics in fixed-point search algorithms. Finally, in Sect. IV] we summarise the main results and comment on their possible relations to classical efficient simulatability of Grover's algorithm.

\section{ENTANGLEMENT DYNAMICS IN GROVER'S ALGORITHM}

Let us remind the reader that the Grover search algorithm [6] employs pure states of $n$ qubits which are initially prepared in an equally weighted superposition of all computational basis states $\left|\psi_{0}\right\rangle=\frac{1}{\sqrt{2^{n}}} \sum_{x=0}^{2^{n}-1}|x\rangle$, which can be more conveniently written as

$$
\left|\psi_{0}\right\rangle=\sqrt{\frac{N-M}{N}}\left|X_{0}\right\rangle+\sqrt{\frac{M}{N}}\left|X_{1}\right\rangle,
$$

where $N=2^{n}$ and $M$ is the number of searched items (in the following also referred to as "solutions" of the search problem). Here, $\left|X_{1}\right\rangle=\frac{1}{\sqrt{M}} \sum_{x_{s}}\left|x_{s}\right\rangle$ represents the superposition of all the states $\left|x_{s}\right\rangle$ that are solutions (i.e. searched items), and $\left|X_{0}\right\rangle=\frac{1}{\sqrt{N-M}} \sum_{x_{n}}\left|x_{n}\right\rangle$ denotes the superposition of all the states $\left|x_{n}\right\rangle$ that are not searched for. The global state after $k$ iterations of the Grover operation $G$ has the form [11, 12]

$$
\left|\psi_{k}\right\rangle \equiv G^{k}\left|\psi_{0}\right\rangle=\cos \theta_{k}\left|X_{0}\right\rangle+\sin \theta_{k}\left|X_{1}\right\rangle
$$

with $\theta_{k}=(k+1 / 2) \theta$ and $\theta=2 \sqrt{M / N}$ in the limit $M \ll$ $N$. The unitary operation $G$ is usually decomposed in two basic blocks, $G=\mathcal{I} U$, where $U$ represents the oracle call, i.e. $U=1-2\left|X_{1}\right\rangle\left\langle X_{1}\right|$, and $\mathcal{I}$ is the inversion about the mean operation, namely $\mathcal{I}=-\left(\mathbf{1}-2\left|\psi_{0}\right\rangle\left\langle\psi_{0}\right|\right)$. The operation $G$ is repeated until the state $\left|\psi_{k}\right\rangle$ overlaps as much as possible with $\left|X_{1}\right\rangle$, namely for $k_{\text {opt }}=C I[(\pi / \theta-$ $1) / 2]$, where $C I[x]$ denotes the closest integer to $x$. In the limit $M \ll N$, the optimal number of iterations is $k_{\text {opt }}=C I\left[\frac{\pi}{4} \sqrt{N / M}-\frac{1}{2}\right]$, i.e. it is proportional to the square root of $N$. In the following we will consider the condition $M \ll N$ to be always fulfilled.

We will now study the entanglement properties of the states $(2)$ as functions of the number of iterations $k$ and the number of qubits $n$ for a fixed number of solutions. We will quantify the amount of entanglement by the GME [13], which for a pure $n$-partite state $|\psi\rangle$ reads

$$
E_{q}(|\psi\rangle)=1-\max _{|\phi\rangle \in S_{q}}|\langle\psi \mid \phi\rangle|^{2}
$$


where $S_{q}$ is the set of $q$-separable states, namely states that are separable for $q$ partitions of the $n$-qubit system. The GME represents a suitable entanglement measure when multi-partite systems are taken into account. Notice that $E_{n}$ quantifies the amount of entanglement of any kind contained in the global system, i.e. it is nonvanishing even for states showing entanglement just between two subsystems, while $E_{2}$ quantifies genuine multipartite entanglement [14.

\section{A. Single solution}

Let us first consider the case of a single solution to the search problem $(M=1)$. W.l.o.g., as will be proved later, we consider the state $\left|X_{1}\right\rangle$ representing the solution to be invariant under any permutation of the $n$ qubits (e.g. $|111 \ldots 1\rangle$ ). Therefore, the state $\left|\psi_{k, M=1}\right\rangle$ at step $k$ of the algorithm is also permutation invariant for all $k$ 's. Let us first compute $E_{n}$ for this set of states for varying $k$. Due to this symmetry property, the search for the maximum in Eq. (3) can be restricted to symmetric separable states $|\phi\rangle^{\otimes n}$ [15, so that the maximisation involves only the two parameters $\alpha \in[0, \pi]$ and $\beta \in[0,2 \pi]$ that define the single qubit state $|\phi\rangle=\cos \frac{\alpha}{2}|0\rangle+e^{i \beta} \sin \frac{\alpha}{2}|1\rangle$. Furthermore, since $\theta_{k} \in[0, \pi / 2]$ the coefficients of $\left|\psi_{k}\right\rangle$ are all positive and the optimal value of the phase factor can be fixed to $\beta=0$.

The GME $E_{n}$ for a single solution then takes the form

$$
\begin{aligned}
E_{n}\left(\left|\psi_{k, M=1}\right\rangle\right)= & 1-\max _{\alpha} \mid \frac{\cos \theta_{k}}{\sqrt{2^{n}-1}}\left[\left(\cos \frac{\alpha}{2}+\sin \frac{\alpha}{2}\right)^{n}\right. \\
& \left.-\sin ^{n} \frac{\alpha}{2}\right]+\left.\sin \theta_{k} \sin ^{n} \frac{\alpha}{2}\right|^{2}
\end{aligned}
$$

The optimal value of $\alpha$ can then be found by setting $t=\tan \frac{\alpha}{2}$ and calculating the derivative of the overlap explicitly, which reduces to finding the root of a polynomial in $t$.

In Fig. 1 we report the behaviour of $E_{n}\left(\left|\psi_{k, M=1}\right\rangle\right)$ for $n=12$ : The entanglement increases in the first half of iterations, achieves the maximal value of about $1 / 2$, and then decreases to zero as soon as the optimal number of iterations is reached. This behaviour is qualitatively similar to the ones shown in 7, , 8 , where the dynamics of both the two-qubit concurrence and the Von Neumann entropy of the half-qubit reduced state was studied.

In order to quantify only genuine multipartite entanglement we will now calculate $E_{2}$. The expression of $E_{2}(|\psi\rangle)$ can be rewritten as [16]

$$
E_{2}(|\psi\rangle)=1-\max _{P} \max _{\mu} \mu^{2}
$$

where the $\mu$ 's are the Schmidt coefficients of $|\psi\rangle$ with respect to a fixed bipartition $P: Q$, and $\max _{P}$ denotes the maximisation over all possible bipartitions. Notice that, since the regarded state $\left|\psi_{k, M=1}\right\rangle$ is permutation invariant, we need to check only $\lfloor n / 2\rfloor$ bipartitions, where $\lfloor x\rfloor$ is the largest integer smaller or equal to $x$. In order to

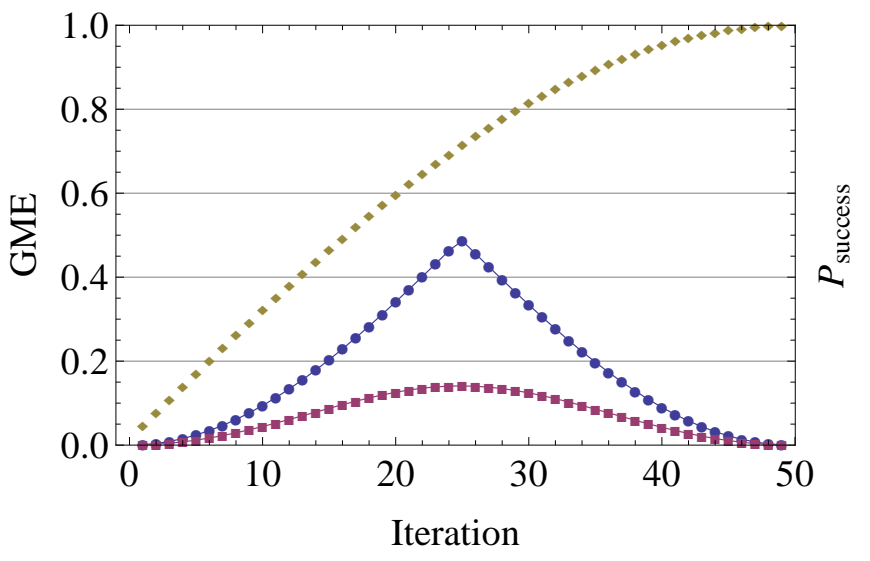

Figure 1. (Color online) Evolution of entanglement as a function of the number of steps $k$, for a single searched item, with $n=12$ qubits and $k_{o p t}=49 . E_{n}\left(\left|\psi_{k, M=1}\right\rangle\right)$ is depicted by blue dots, while $E_{2}\left(\left|\psi_{k, M=1}\right\rangle\right)$ by purple squares. The yellow dots represent the success probability.

find the maximal Schmidt coefficient of $\left|\psi_{k, M=1}\right\rangle$ among all possible bipartitions we fix a generic bipartite splitting $P: Q$, where $P$ is composed of $m$ qubits and $Q$ of the remaining $n-m$, and compute the eigenvalues of the reduced density operator $\rho_{P}=\operatorname{Tr}_{Q}\left[\left|\psi_{k, M=1}\right\rangle\left\langle\psi_{k, M=1}\right|\right]$, given by the following $2^{m} \times 2^{m}$ matrix

$$
\rho_{P}=\left(\begin{array}{cccc}
a & \ldots & a & b \\
\vdots & \ddots & \vdots & \vdots \\
a & \ldots & a & b \\
b & \ldots & b & c
\end{array}\right),
$$

where $a=2^{n-m} A^{2}, b=a-A(A-B)$, and $c=a-$ $A^{2}+B^{2}$, with $A=\cos \theta_{k} / \sqrt{2^{n}-1}$ and $B=\sin \theta_{k}$. The maximal eigenvalue of the above matrix is given by

$$
\lambda_{\max }=\frac{1}{2}+\frac{1}{2}\left[1-4\left(2^{m}-1\right)\left(2^{n-m}-1\right) A^{2}(A-B)^{2}\right]^{\frac{1}{2}} .
$$

The above expression shows that the bipartition that leads to the maximum eigenvalue corresponds to $m=1$ for all values of $k$. According to Eq. (5), the multipartite GME $E_{2}$ takes the explicit form

$$
\begin{aligned}
& E_{2}\left(\left|\psi_{k, M=1}\right\rangle\right)= \\
& \quad \frac{1}{2}-\frac{1}{2}\left[1-4 \frac{2^{n-1}-1}{2^{n}-1} \cos ^{2} \theta_{k}\left(\frac{\cos \theta_{k}}{\sqrt{2^{n}-1}}-\sin \theta_{k}\right)^{2}\right]^{\frac{1}{2}} .
\end{aligned}
$$

This result shows that genuine multipartite entanglement has a qualitative similar behaviour as $E_{n}\left(\left|\psi_{k, M=1}\right\rangle\right)$ (see Fig. 1), even if it achieves a maximum of about 0.14 and the curve is derivable in that point. Notice also that $E_{2}\left(\left|\psi_{k, M=1}\right\rangle\right)$ is symmetric with respect to $k_{\text {opt }} / 2$.

We will now show that the entanglement dynamics in the Grover algorithm, namely the behaviour of $E_{n}$ and 
$E_{2}$ during the operation of the algorithm, does not depend on the number of qubits $n$, thus exhibiting the property of scale invariance. For $2^{n} \gg 1$ the two entanglement measures take the simple forms

$$
\begin{aligned}
& E_{n}\left(\left|\psi_{k, M=1}\right\rangle\right) \simeq \begin{cases}\sin ^{2} \theta_{k} & \text { for } \theta_{k} \leq \pi / 4, \\
\cos ^{2} \theta_{k} & \text { for } \theta_{k}>\pi / 4,\end{cases} \\
& E_{2}\left(\left|\psi_{k, M=1}\right\rangle\right) \simeq \frac{1}{2}\left[1-\left(1-\frac{1}{2} \sin ^{2} 2 \theta_{k}\right)^{\frac{1}{2}}\right] .
\end{aligned}
$$

Both expressions depend only on $\theta_{k} \simeq \frac{\pi}{2} k / k_{\text {opt }}$, namely on $k / k_{o p t}$, and not on $k$ and $n$ separately. Therefore, the entanglement dynamics of the Grover algorithm is scale invariant in the sense that it only depends on the number of steps taken, relative to the total number, but not on the length of the list.

We want to point out that all the results presented so far, even if they were explicitly derived for permutation invariant states, hold for any instance of the Grover algorithm with one searched item, i.e. $M=1$. The number of possible single searched items in the Grover algorithm is $2^{n}$, which corresponds to the number of distinct states $\left|X_{1}\right\rangle$. All of these states can be achieved from a symmetric one by applying tensor products of $\sigma_{x}$ Pauli operators and identity operators 1 (e.g. $|001 \ldots 1\rangle=$ $\left.\sigma_{x 1} \otimes \sigma_{x 2} \otimes \mathbf{1}_{3} \ldots .|111 \ldots 1\rangle\right)$. Since these operations are local, they do not change the entanglement content of the resulting state.

\section{B. Two solutions}

Let us now consider the case of two searched items (i.e. $M=2)$. As an illustrative example we will consider the case in which both $|00 \ldots 0\rangle$ and $|11 \ldots 1\rangle$ are solutions of the search problem, thus the state $\left|X_{1}\right\rangle$ is a GHZ state composed of $n$ qubits, and the state at each step of the computation is permutation invariant. The measure of any entanglement $E_{n}$ is given by

$$
\begin{aligned}
& E_{n}\left(\left|\psi_{k, M=2}\right\rangle\right)=1-\max _{\alpha} \mid \frac{\cos \theta_{k}}{\sqrt{2^{n}-2}}\left[\left(\cos \frac{\alpha}{2}+\sin \frac{\alpha}{2}\right)^{n}\right. \\
& \left.-\left(\cos ^{n} \frac{\alpha}{2}+\sin ^{n} \frac{\alpha}{2}\right)\right]+\left.\frac{\sin \theta_{k}}{\sqrt{2}}\left(\cos ^{n} \frac{\alpha}{2}+\sin ^{n} \frac{\alpha}{2}\right)\right|^{2},
\end{aligned}
$$

We maximised this quantity numerically; in Fig. 2 we show the behavior for $n=13$. Notice that after $k_{\text {opt }}$ iterations, the measure $E_{n}\left(\left|\psi_{k, M=2}\right\rangle\right)$ is no longer zero but equal to $1 / 2$. That is because the final state is no longer fully separable but instead it is close to the GHZ state. In this case the maximal value that the entanglement reaches during the computation is about $2 / 3$, i.e. higher than the case $M=1$. Furthermore, this maximum is no longer reached at half of the optimal number of steps $k_{\text {opt }}$, but in a later step, i.e. $k / k_{\text {opt }} \simeq 0.61$.

Regarding genuine multipartite entanglement, $E_{2}$ with two symmetric solutions can be computed by following an

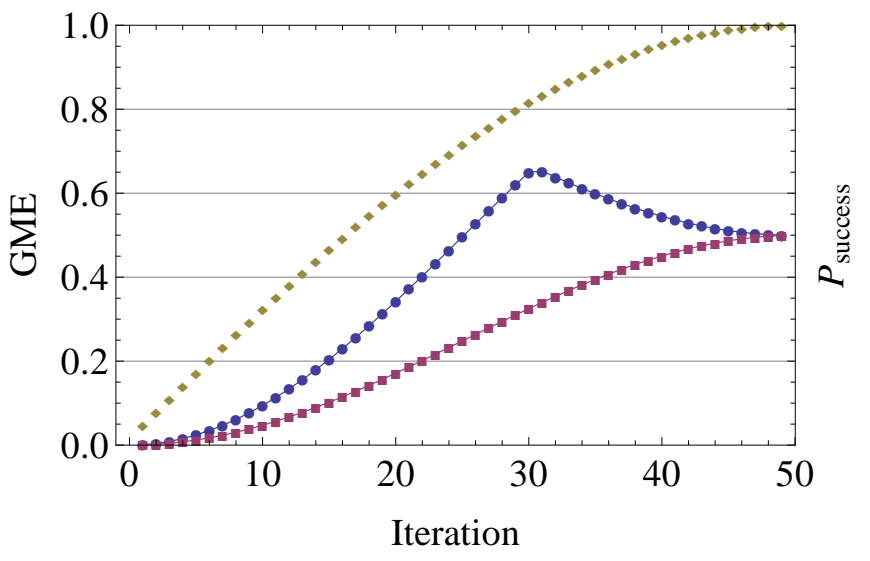

Figure 2. (Color online) The GME as a function of the number of steps $k$, for two symmetric solutions of the search problem. The number of qubits is $n=13$, and $k_{\text {opt }}=49 . E_{n}\left(\left|\psi_{k, M=2}\right\rangle\right)$ is given by blue dots, $E_{2}\left(\left|\psi_{k, M=2}\right\rangle\right)$ by purple squares. The yellow dots represent the success probability.

analogous procedure as for a single solution. The reduced density matrix for the general bipartite splitting $P: Q$, where $m$ qubits are in $P$ and $n-m$ in $Q$, is now given by

$$
\rho_{P}=\left(\begin{array}{ccccc}
c & b & \ldots & b & d \\
b & a & \ldots & a & b \\
\vdots & \vdots & \ddots & \vdots & \vdots \\
b & a & \ldots & a & b \\
d & b & \ldots & b & c
\end{array}\right)
$$

where $a, b, c$ and $A, B$ are given below Eq. (6), and $d=a-2 A(A-B)$. It turns out that again the maximum eigenvalue corresponds to the bipartite splitting with $m=1$, and $E_{2}\left(\left|\psi_{k, M=2}\right\rangle\right)$ can be expressed analytically as

$$
E_{2}\left(\left|\psi_{k, M=2}\right\rangle\right)=1-\frac{2^{n}-4}{2^{n}-2} \cos ^{2} \theta_{k}-\left(\frac{\cos \theta_{k}}{\sqrt{2^{n}-2}}+\frac{\sin \theta_{k}}{\sqrt{2}}\right)^{2} .
$$

This result is shown in Fig. 2. Notice that multipartite entanglement has a different behaviour from $E_{n}\left(\left|\psi_{k, M=2}\right\rangle\right)$. It is a monotonically increasing function that approaches the maximum value of $1 / 2$ when the computation stops.

In the asymptotic limit $2^{n} \gg 1$ the GME can be expressed as

$$
\begin{aligned}
& E_{n}\left(\left|\psi_{k, M=2}\right\rangle\right) \simeq \begin{cases}\sin ^{2} \theta_{k} & \text { for } \theta_{k} \leq \arccos 1 / \sqrt{3}, \\
\frac{1+\cos ^{2} \theta_{k}}{2} & \text { for } \theta_{k}>\arccos 1 / \sqrt{3},\end{cases} \\
& E_{2}\left(\left|\psi_{k, M=2}\right\rangle\right) \simeq \frac{1}{2} \sin ^{2} \theta_{k} .
\end{aligned}
$$

As a consequence, both quantities exhibit the same scale invariance behaviour as discussed above for the case with one searched item, i.e. $M=1$. We point out that the 


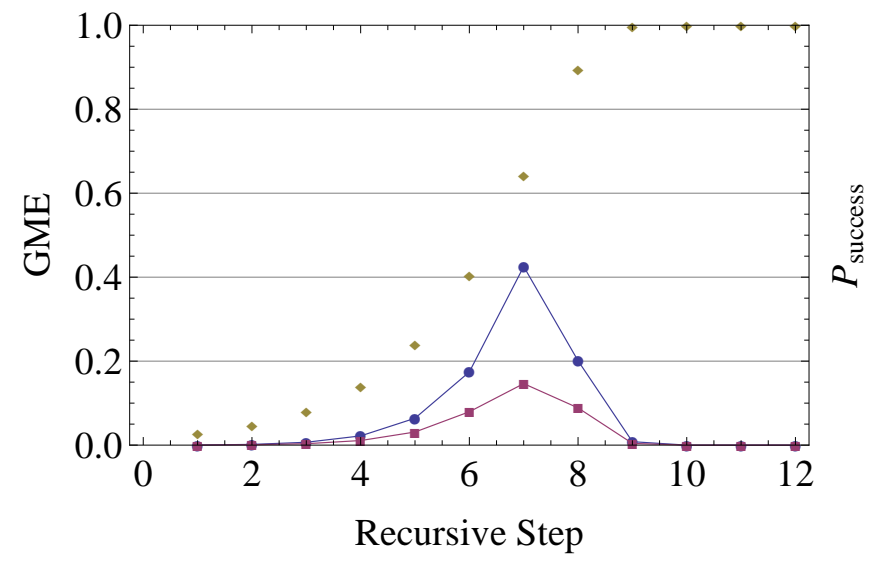

Figure 3. (Color online) Evolution of entanglement in the $\pi / 3$ search as a function of the recursive step $m$, for a single searched item, with $n=12$ qubits. Here and below in Fig. 4 blue dots, purple squares and yellow dots denote $E_{n}, E_{2}$ and the success probability, respectively.

above results can be generalized to those search problems in which the two solutions are different in all digits, but not to all search problems with $M=2$.

\section{ENTANGLEMENT DYNAMICS IN THE FIXED-POINT $\pi / 3$ QUANTUM SEARCH ALGORITHM}

In the previous section we have shown that the amount of entanglement is non-vanishing during the Grover algorithm and that its behaviour is scale invariant for a single solution to the search problem and in some instances of two solutions. We will now show that a similar entanglement dynamics can be found in the fixed-point $\pi / 3$ quantum search. This kind of quantum search algorithm was first introduced in [9] to overcome the fact that the Grover algorithm might lead to a high error probability if the number of solutions $M$ is unknown, since it requires to stop at a precise iteration $k_{o p t}$, which depends on $M$. In contrast the $\pi / 3$ quantum search always converges to the solutions, and thus it can be regarded as a fixed-point algorithm, even if it is never as fast as the standard Grover algorithm.

A possible way to realise such a fixed-point search is to slightly modify the operations $U$ and $\mathcal{I}$ in order to produce a $\pi / 3$ shift instead of a $\pi$ shift [9], i.e.

$$
\begin{aligned}
& U \longrightarrow U_{\frac{\pi}{3}}=\mathbf{1}-\left(1-e^{i \frac{\pi}{3}}\right)\left|X_{1}\right\rangle\left\langle X_{1}\right|, \\
& \mathcal{I} \longrightarrow \mathcal{I}_{\frac{\pi}{3}}=-\left(\mathbf{1}-\left(1-e^{i \frac{\pi}{3}}\right)\left|\psi_{0}\right\rangle\left\langle\psi_{0}\right|\right) .
\end{aligned}
$$

Then, the sequence of gates to be applied is defined by the following recursive formula

$$
\begin{aligned}
& A_{m+1}=A_{m} \mathcal{I}_{\frac{\pi}{3}} A_{m}^{\dagger} U_{\frac{\pi}{3}} A_{m}, \\
& A_{0}=\mathbf{1} .
\end{aligned}
$$

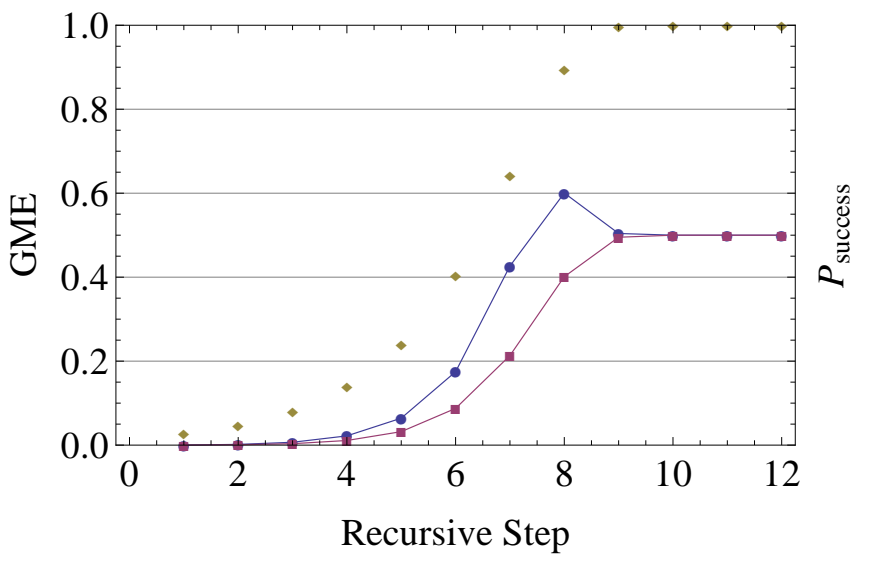

Figure 4. (Color online) Evolution of entanglement in the $\pi / 3$ search as a function of the recursive step $m$, for two symmetric solutions of the search problem, with $n=13$ qubits.

We now compute both $E_{n}$ and $E_{2}$ for the employed states at each recursive step $m$ of the evolution. The results were obtained numerically and are shown in Figs. 3 and 4 for both one and two solutions. Notice that the entanglement behaviour of both $E_{n}$ and $E_{2}$ is similar to the dynamics of the standard Grover algorithm. These results indicate that entanglement plays the same crucial role in both algorithms, even if a scale invariance property cannot be proved in the fixed-point algorithm case.

\section{CONCLUSIONS}

In summary, we have studied the evolution of entanglement in Grover's algorithm (for a small number of searched items), quantifying it via the GME. In particular, we give an explicit formula for the amount of genuine multipartite entanglement, which is proven to be always non-zero throughout the computation. Interestingly, the dynamics of entanglement shows the behaviour of scale invariance, i.e. counter-intuitively the amount of entanglement employed in the algorithm does not depend on the length of the searched list, but only on the number of steps taken, relative to the optimal number of steps. Since scale invariance is an important phenomenon in several areas of physics and mathematics, our results may open new avenues in the understanding of scale invariance properties of entanglement in other contexts, such as for example in many-body systems and phase transitions. We have also compared the Grover search entanglement dynamics with the one of a different kind of search algorithm, i.e. the $\pi / 3$ quantum search, and we have showed that they exhibit a similar behavior.

As a final comment, we may wonder whether the presence of true multipartite entanglement means that Grover's algorithm cannot be simulated efficiently by classical means. By efficient classical simulation of Grover's algorithm we mean that, given a database of 
$n$ qubits, i.e. $2^{n}$ items, it is possible to classically simulate Grover's algorithm with a total cost that scales as $\sqrt{2^{n}}$ poly $(n)$. We will now show that well-known criteria which guarantee efficient simulatability do not apply for Grover's algorithm. According to the GottesmanKnill theorem [2, 12], if a quantum computation starts in a computational basis state and involves only stabilizer gates (i.e. Hadamard, CNOT, phase gates and measurement of operators in the Pauli group), then it can be efficiently simulated on a classical computer. However, it can be easily shown that $\mathcal{I}$ transforms an element belonging to the Pauli group, i.e. $\sigma_{z} \otimes \mathbf{1}^{(n-1)}$, to an operator that no longer belongs to the Pauli group, and therefore it cannot be implemented by stabilizer gates. Let us also consider the simulatability criterion introduced in [3, based on the maximal Schmidt rank $\chi$ of $|\psi\rangle$ over all possible bipartitions. According to [3], if $\chi$ does not exceed $\operatorname{poly}(n)$ in a computation that consists of $\operatorname{poly}(n)$ elementary gates (i.e. one- and two-qubit gates), then the computation can be classically simulated efficiently. We notice that for states of the form (2), $\chi$ is upper bounded by $M+1$. However, although there exists a decomposition of the Grover operation $G$ into poly $(n)$ elementary gates [12, 17, the state after the action of each two-qubit gate does not have a simple symmetric form and we no longer can keep track of the maximal Schmidt rank. Therefore we cannot conclude efficient simulatability.

The above results show that, although the Grover operation cannot be implemented by stabilizer gates and therefore the Knill-Gottesman theorem cannot be applied, the employed states at each Grover iteration are only slightly entangled according to the criterion suggested in 3 . These insights are nevertheless not sufficient to answer the question of simulatability of the Grover algorithm, which at present remains open.

\section{ACKNOWLEDGEMENTS}

This work was financially supported by DFG. MR is supported by DAAD and acknowledges the hospitality of the Heinrich-Heine Universität Düsseldorf.
[1] R. Jozsa and N. Linden, Proc. R. Soc. Lond. A 459, 2011 (2003).

[2] S. Aaronson and D. Gottesman, Phys. Rev. A 70, 052328 (2004).

[3] G. Vidal, Phys. Rev. Lett. 91, 147902 (2003).

[4] M. Van den Nest, preprint quant-ph/1204.3107 (2012).

[5] D. Bruß and C. Macchiavello, Phys. Rev. A 83, 052313 (2011).

[6] L.K. Grover, in Proceeding of the 28th Annual Symposium on the Theory of Computing (ACM Press, New York, 1996), pp. 212-219; L.K. Grover, Phys. Rev. Lett. 79, 325 (1997).

[7] Y. Fang et al., Phys. Lett. A 345, 265 (2005).

[8] R. Orús and J.I. Latorre, Phys. Rev. A 69, 052308 (2004).

[9] L.K. Grover, Phys. Rev. Lett. 95, 150501 (2005).
[10] T. Tulsi, L.K. Grover and A.Patel, Quant. Inf. Comput. 6,483 (2006).

[11] D. Aharonov. Quantum Computation. In D. Stauffer, editor, Annual Reviews of Computational Physics VI, World Scientific, Singapore, 1999.

[12] M.A. Nielsen and I.L. Chuang, Quantum computation and quantum information, Cambridge University Press, Cambridge U.K., 2000.

[13] T.-C. Wei and P.M. Goldbart, Phys. Rev. A 68, 042307 (2003).

[14] M. Blasone, F. Dell'Anno, S. De Siena and F. Illuminati, Phys. Rev. A 77, 062304 (2008).

[15] R. Hübener, M. Kleinmann, T.-C. Wei, C. GonzálezGuillén, and O. Gühne, Phys. Rev. A 80, 032324 (2009).

[16] M. Bourennane et al., Phys. Rev. Lett. 92, 087902 (2004).

[17] Z. Diao, M.S. Zubairy, and G. Chen, Z. Naturforsch. 57a, 701 (2002). 